\title{
Bohmian Quantum Gravity in the Linear Field Approximation
}

ALI SHOJAI*

Physics Department, Tehran University, End of North Karegar St., Tehran 14352, IRAN

and

Institute for Studies in Theoretical Physics and Mathematics, P.O.Box 19395-5531, Tehran, $\operatorname{IRAN}$

\section{FATIMAH SHOJAI ${ }^{\dagger}$}

Physics Department, Iran University of Science and Technology, P.O.Box 16765-163, Narmak, Tehran, IRAN

and

Institute for Studies in Theoretical Physics and Mathematics, P.O.Box 19395-5531, Tehran, IRAN

In this paper we have applied Bohmian quantum theory to the linear field approximation of gravity and present a self-consistent quantum gravity theory in the linear field approximation. The theory is then applied to some specific problems, the Newtonian limit, and the static spherically symmetric solution. Some observable effects of the theory are investigated.

PACS NO.: 03.65.Ta ; 04.60.-m

\footnotetext{
*Email: SHOJAI@IPM.IR

${ }^{\dagger}$ Email: FATIMAH@IPM.IR
} 


\section{INTRODUCTION}

There are several approaches to the quantization of gravity. Some important ones are, the canonical formalism (or the WDW formalism) [1], Narlikar and Padmanabhan approach to quantization of the conformal degree of freedom of the space-time metric [2], the standard Bohmian quantum gravity [3,4], and a new approach [5-7] relating quantum mechanics and the geometry of the space-time. Each of the above theories have some positive and some negative aspects, so that there is no universally accepted theory of quantum gravity. As some authors have mentioned, Bohmian quantum gravity has some noticable aspects. It has not the problem of time, the measurement problem and the difficulty with the meaning of the wavefunction [8]. Also it presents a trajectory for the system just like in the classical domain [3].

To understand how this theory works, let us briefly explain the Bohmian quantum me-

chanics of a single non-relativistic particle [9]. In the standard quantum mechanics, the particle's wavefunction satisfies the Schrödinger equation:

$$
i \hbar \frac{\partial \Psi}{\partial t}=-\frac{\hbar^{2}}{2 m} \nabla^{2} \Psi+V(\vec{x} ; t) \Psi
$$

If one decomposes the wavefunction into its norm and its phase:

$$
\Psi=R \exp (i S / \hbar)
$$

one gets the following two equations:

$$
\begin{aligned}
& \frac{\partial S}{\partial t}+\frac{|\vec{\nabla} S|^{2}}{2 m}+V+\mathcal{Q}=0 \\
& \frac{\partial R^{2}}{\partial t}+\vec{\nabla} \cdot\left(R^{2} \frac{\vec{\nabla} S}{m}\right)=0
\end{aligned}
$$


where

$$
\mathcal{Q}=-\frac{\hbar^{2}}{2 m} \frac{\nabla^{2} R}{R}
$$

As it can be seen, we have a modified Hamilton-Jacobi equation and the continuity equation provided we assume that the particle's trajectory is given by the classical relation:

$$
\vec{v}=\frac{\vec{\nabla} S}{m}
$$

The Newton's equation of motion can be derived by taking the gradiant of the HamiltonJacobi equation and using the guidance relation (6). The result is:

$$
m \frac{d^{2} \vec{x}}{d t^{2}}=-\vec{\nabla} V-\vec{\nabla} \mathcal{Q}
$$

So the Bohmian interpretation is as follows. To quantize any classical system add a quantum potential to the classical Hamilton-Jacobi equation. The quantum potential is given in terms of the density of an hypothetical ensemble of the system $\left(R^{2}\right)$ and thus add the continuity equation to have a self-consistent system of equations.

Bohmian quantum gravity is also constructed successfully [4,3]. But it has also some problems. The most essential one is perhaps the lack of general covariance [10]. Although some authors look at this property as an essential character of quantum gravity [11].

Investigating Bohmian quantum gravity in the linear field approximation of gravity is perhaps a good idea, because as it is the case for classical gravity some important effects can be understood better, in the linear approximation. In this paper we shall do this and then study the theory for the Newtonian limit and the static spherically symmetric metric, and some observable phenomena are predicted. 


\section{LINEAR FIELD BOHMIAN QUANTUM GRAVITY}

Our plan in this section is to make Bohmian quantum gravity in the linear field approximation, i.e. when the space-time metric is expanded about the flat Minkowski space-time as:

$$
g_{\mu \nu}=\eta_{\mu \nu}+h_{\mu \nu}
$$

and considering only linear terms in $h_{\mu \nu}$. The lagrangian density of the linear field classical gravity is given by [12]:

$$
\mathcal{L}=\frac{1}{2} \partial_{\lambda} \phi_{\mu \nu} \partial^{\lambda} \phi^{\mu \nu}
$$

where as it is convenient we introduced the new field variables:

$$
\phi_{\mu \nu}=h_{\mu \nu}-\frac{1}{2} h \eta_{\mu \nu}
$$

The canonical momenta are defined as:

$$
\Pi_{\mu \nu}=\frac{\delta \mathcal{L}}{\delta \dot{\phi}^{\mu \nu}}=\frac{\partial \phi_{\mu \nu}}{\partial t}
$$

and the hamiltonian density is:

$$
\mathcal{H}=\frac{1}{2} \Pi_{\mu \nu} \Pi^{\mu \nu}+\frac{1}{2} \nabla_{i} \phi_{\mu \nu} \nabla^{i} \phi^{\mu \nu}
$$

The general coordinate transformation invariance of the linear field gravity is guaranteed by choosing some specific gauge. The common gauge is the harmonic gauge:

$$
\partial^{\mu} \phi_{\mu \nu}=0
$$

The transition to the quantum theory can be achieved via Dirac's canonical quantization scheme, in which we replace: 


$$
\Pi_{\mu \nu} \rightarrow-i \hbar \frac{\delta}{\delta \phi_{\mu \nu}}
$$

leading to the following wave equation:

$$
i \hbar \frac{\partial \Psi}{\partial t}=\left(-\frac{\hbar^{2}}{2} \int d^{3} x^{\prime} \frac{\delta^{2}}{\delta \phi_{\mu \nu}\left(x^{\prime}\right) \delta \phi^{\mu \nu}\left(x^{\prime}\right)}+\frac{1}{2} \int d^{3} x^{\prime} \nabla_{i}^{\prime} \phi_{\mu \nu} \nabla^{\prime i} \phi^{\mu \nu}\right) \Psi
$$

where $\Psi\left[\phi_{\alpha \beta}\right]$ is the wavefunctional.

The Bohmian interpretation of this wave equation can be achieved via setting:

$$
\Psi=R \exp (i S / \hbar)
$$

leading to the Hamilton-Jacobi equation:

$$
\frac{\partial S}{\partial t}+\frac{1}{2} \int d^{3} x^{\prime} \frac{\delta S}{\delta \phi_{\mu \nu}\left(x^{\prime}\right)} \frac{\delta S}{\delta \phi^{\mu \nu}\left(x^{\prime}\right)}+\frac{1}{2} \int d^{3} x^{\prime} \nabla_{i}^{\prime} \phi_{\mu \nu}\left(x^{\prime}\right) \nabla^{i} \phi^{\mu \nu}\left(x^{\prime}\right)+\mathcal{Q}=0
$$

and the continuity equation:

$$
\frac{\partial R^{2}}{\partial t}+\int d^{3} x^{\prime} \frac{\delta}{\delta \phi_{\mu \nu}\left(x^{\prime}\right)}\left(R^{2} \frac{\delta S}{\delta \phi^{\mu \nu}\left(x^{\prime}\right)}\right)=0
$$

with the quantum potential defined as:

$$
\mathcal{Q}=-\frac{\hbar^{2}}{2 R} \int d^{3} x^{\prime} \frac{\delta^{2} R}{\delta \phi_{\mu \nu}\left(x^{\prime}\right) \delta \phi^{\mu \nu}\left(x^{\prime}\right)}
$$

The Bohmian trajectories can be obtained via the guidance equation:

$$
\frac{\partial \phi_{\mu \nu}}{\partial t}=\frac{\delta S}{\delta \phi^{\mu \nu}}
$$

and the gauge condition is given by the equation (13).

It is important here to note that we have not included the gauge condition as it is always done. That is to say we have not set:

$$
\partial_{\mu} \phi^{\mu \nu}=0 \Rightarrow\left(\Pi^{0 \nu}+\partial_{i} \phi^{i \nu}\right)|\Psi\rangle=\left(-i \hbar \frac{\delta}{\delta \phi_{0 \nu}}+\partial_{i} \phi^{i \nu}\right)|\Psi\rangle=0
$$


Because this forces the state to satisfy the gauge condition and the Bohmian trajectories are not necessarily consistent with it. If one wishes to save the gauge condition at the level of trajectories, one should apply it on the Bohmian trajectories $\phi^{\mu \nu}(\vec{x}, t)$.

The field equation can be derived by taking variation of the Hamilton-Jacobi equation (17) with respect to $\phi_{\mu \nu}$. The result is:

$$
\square \phi_{\mu \nu}(x)=-\frac{\delta \mathcal{Q}}{\delta \phi^{\mu \nu}(x)}
$$

Let us now, apply these results to the Newtonian approximation. In this limit the metric is given by:

$$
h_{00}=h=2 \Phi ; \quad h_{i j}=h_{0 i}=0
$$

where $\Phi$ is the Newton's gravitational potential. The $\phi$-field is given by:

$$
\phi_{00}=\Phi ; \quad \phi_{i j}=\Phi \delta_{i j} ; \quad \phi_{0 i}=0
$$

and we have the identities:

$$
\frac{\delta}{\delta \phi_{00}}=\frac{\delta}{\delta \Phi} ; \quad \frac{\delta}{\delta \phi_{i j}}=\delta_{i j} \frac{\delta}{\delta \Phi}
$$

So the Hamilton-Jacobi equation and the continuity equation are given by:

$$
\begin{gathered}
\frac{\partial S}{\partial t}+\int d^{3} x^{\prime}\left(\frac{\delta S}{\delta \Phi\left(x^{\prime}\right)}\right)^{2}+\frac{1}{2} \int d^{3} x^{\prime} \nabla_{i}^{\prime} \Phi\left(x^{\prime}\right) \nabla^{\prime i} \Phi\left(x^{\prime}\right)+\mathcal{Q}=0 \\
\frac{\partial R^{2}}{\partial t}+4 \int d^{3} x^{\prime} \frac{\delta}{\delta \Phi\left(x^{\prime}\right)}\left(R^{2} \frac{\delta S}{\delta \Phi\left(x^{\prime}\right)}\right)=0
\end{gathered}
$$

where the quantum potential is given by:

$$
\mathcal{Q}=-\frac{2 \hbar^{2}}{R} \int d^{3} x^{\prime} \frac{\delta^{2} R}{\delta \Phi\left(x^{\prime}\right)^{2}}
$$


The guidance relation is:

$$
\frac{\partial \Phi}{\partial t}=\frac{\delta S}{\delta \Phi}
$$

and the condition of the Newtonian approximation is given by:

$$
\frac{\partial \Phi}{\partial t}=0
$$

instead of the relation (13). As a result of this last equation we have:

$$
\frac{\partial \Phi}{\partial t}=\frac{\delta S}{\delta \Phi}=0
$$

so we have encountered with a pure quantum case $^{1}$ and so we have:

$$
-E+\frac{1}{2} \int d^{3} x^{\prime} \nabla_{i}^{\prime} \Phi\left(x^{\prime}\right) \nabla^{\prime i} \Phi\left(x^{\prime}\right)=\mathcal{Q}
$$

where we have assumed $\partial S / \partial t=-E$, because in the Newtonian limit physical quantities should not depend on time. The continuity equation is satisfied identically.

The field equation can be derived by differentiating the Hamilton-Jacobi equation with respect to $\Phi$. The result is:

$$
\nabla^{2} \Phi=\frac{\delta \mathcal{Q}}{\delta \Phi(x)}
$$

Until now we have encountered with the free linear field Bohmian quantum gravity. That is we have not considered the matter. If one wants to add the matter field semi-classically ${ }^{2}$,

\footnotetext{
${ }^{1}$ That is a case in which quantum potential balances the classical potential. So the quantum potential is not ignorable and we have no classical limit [3].

${ }^{2}$ In order to take into account the quantum effects of matter, one should start from the linear field
} 
one should add merely the source term $-\kappa \rho$ to the above equation, where $\rho$ is the matter density and $\kappa$ is the gravitational constant. So we have:

$$
\nabla^{2} \Phi=-\kappa \rho+\frac{\delta \mathcal{Q}}{\delta \Phi(x)}
$$

\section{STATIC SPHERICALLY SYMMETRIC SOLUTION}

Let's now look for static spherically symmetric solution of the equations of the previous section. We do this in the case of Newtonian and weak field approximations.

(a) First we work in the Newtonian approximation. Let's choose $R$ as a packet around the classical solution of the gravitational field equation:

$$
R=\exp \left\{-\frac{\alpha}{2}\left[\Phi(x)-\Phi^{(c)}(x)\right]^{2}\right\}
$$

where $\Phi^{(c)}$ is the classical field, i.e. the field satisfying the equation (34) without $\mathcal{Q}$.

This leads to the following quantum potential:

$$
\mathcal{Q}=-\alpha \hbar^{2}\left[1-\alpha\left(\Phi-\Phi^{(c)}\right)^{2}\right]
$$

so the field equation is given by:

$$
\nabla^{2} \Phi=-\kappa \rho-\alpha^{2} \hbar^{2}\left(\Phi-\Phi^{(c)}\right)
$$

Let us look for the gravitational potential, when we have a point-like source:

lagrangian density with matter, and then proceed as in the previous section. The only difference would be that the matter quantum potential is also present in the field equations. We did not do so here, because in this work, the quantum effects of gravity are of consider. 


$$
\rho=M \delta(\vec{x})
$$

We solve the equation (37) by setting:

$$
\Phi=\Phi^{(c)}+f=-\frac{\kappa M}{r}+f
$$

so we have:

$$
\nabla^{2} f=-\alpha^{2} \hbar^{2} f
$$

The solution is given by:

$$
\Phi=-\frac{\kappa M}{r}+\sum_{l m}\left[a_{l m} j_{l}(\alpha \hbar r)+b_{l m} n_{l}(\alpha \hbar r)\right] Y_{l m}(\theta, \varphi)
$$

where $a_{l m}$ and $b_{l m}$ are constants.

Some points about this solution is of interest:

- Because of the asymptotic behaviour of the spherical Bessel functions:

$$
\begin{aligned}
& j_{l}(x) \sim \frac{1}{x} \sin \left(x-\frac{l \pi}{2}\right) \quad \text { as } x \rightarrow \infty \\
& n_{l}(x) \sim \frac{1}{x} \cos \left(x-\frac{l \pi}{2}\right) \quad \text { as } x \rightarrow \infty
\end{aligned}
$$

one can see that this quantum solution also behaves as $\frac{1}{r}$ at large distances from the source.

- It must be noted that the weak field approximation is only applicable for large $r$ 's. So this solution does not tell anything about the event horizon or about the singularity. 
- If one sets $M=0$, so that we have no source at all, we have a pure quantum solution in which we have source-less gravity. This means that the quantum fluctuations of gravity can produce observable gravity.

- Because of the oscillatory nature of the spherical Bessel functions, the gravitational potential is also oscillatory. So we have a set of periodic stable points, where the potential is minimum, and a test particle can be at rest at that locations (This is true also for the case $M=0$ ). It must be noted that, if we choose a sharp wave packet $(\alpha \rightarrow \infty$ in equation (35)), the frequency of such oscillations is very large.

(b) Let's now investigate the static spherically symmetric metric in the linear field approximation. The relations (17) and (18) would be now:

$$
\frac{1}{2} \int d^{3} x^{\prime} \frac{\delta S}{\delta \phi_{\mu \nu}\left(x^{\prime}\right)} \frac{\delta S}{\delta \phi^{\mu \nu}\left(x^{\prime}\right)}+\frac{1}{2} \int d^{3} x^{\prime} \nabla_{i}^{\prime} \phi_{\mu \nu}\left(x^{\prime}\right) \nabla^{\prime i} \phi^{\mu \nu}\left(x^{\prime}\right)+\mathcal{Q}=0
$$

and

$$
\int d^{3} x^{\prime} \frac{\delta}{\delta \phi_{\mu \nu}\left(x^{\prime}\right)}\left(R^{2} \frac{\delta S}{\delta \phi^{\mu \nu}\left(x^{\prime}\right)}\right)=0
$$

The Bohmian trajectories can be obtained via the guidance equation:

$$
0=\frac{\partial \phi_{\mu \nu}}{\partial t}=\frac{\delta S}{\delta \phi_{\mu \nu}}
$$

and the gauge condition (13). It is a simple task to see that the corrected field equation is given by:

$$
\nabla^{2} \phi_{\mu \nu}=\frac{\delta \mathcal{Q}}{\delta \phi^{\mu \nu}(x)}
$$


As in the Newtonian case, choosing:

$$
R=\exp \left\{-\frac{\alpha}{2}\left[\phi_{\mu \nu}-\phi_{\mu \nu}^{(c)}\right]\left[\phi^{\mu \nu}-\phi^{(c) \mu \nu}\right]\right\}
$$

we have the following relation for the quantum potential:

$$
\mathcal{Q}=\frac{\alpha \hbar^{2}}{2}\left\{4-\alpha\left[\phi_{\mu \nu}-\phi_{\mu \nu}^{(c)}\right]\left[\phi^{\mu \nu}-\phi^{(c) \mu \nu}\right]\right\}
$$

so the field equations are:

$$
\nabla^{2} \phi_{\mu \nu}+\alpha^{2} \hbar^{2}\left[\phi_{\mu \nu}-\phi_{\mu \nu}^{(c)}\right]=0 ; \quad \partial_{\mu} \phi^{\mu \nu}=0
$$

writing:

$$
\phi_{\mu \nu}=\phi_{\mu \nu}^{(c)}+f_{\mu \nu}=\frac{C_{\mu \nu}}{r}+f_{\mu \nu}
$$

where $\phi_{\mu \nu}^{(c)}=\frac{C_{\mu \nu}}{r}$ with $C_{\mu \nu}$ constants, is the classical solution [12]. The field equation leads to:

$$
\nabla^{2} f_{\mu \nu}+\alpha^{2} \hbar^{2} f_{\mu \nu}=0
$$

The above equation can be solved just as in the previous section. Then one must apply the gauge condition. This would leads to some relation for constants $C_{\mu \nu}$. The solution is:

$$
\phi_{\mu \nu}=\left(\begin{array}{ccccc}
-\frac{2 r_{s}}{r}+f & 0 & 0 & 0 \\
0 & 0 & 0 & 0 \\
0 & 0 & 0 & 0 \\
0 & 0 & 0 & 0
\end{array}\right)
$$


where $f(r, \theta, \varphi)$ is just as in the previous section, i.e.:

$$
f(r, \theta, \varphi)=\sum_{l m}\left[a_{l m} j_{l}(\alpha \hbar r)+b_{l m} n_{l}(\alpha \hbar r)\right] Y_{l m}(\theta, \varphi)
$$

and $r_{s}$, the only non vanishing element of $C_{\mu \nu}$, is the Schwarzschild radius. This solution leads to:

$$
\begin{gathered}
h_{00}=-\frac{r_{s}}{r}+\frac{f}{2} \\
h_{0 i}=0 \\
h_{i i}=-\frac{r_{s}}{r}+\frac{f}{2}
\end{gathered}
$$

So the metric $g_{\mu \nu}=\eta_{\mu \nu}+h_{\mu \nu}$ is given by:

$$
g_{\mu \nu}=\left(\begin{array}{cccc}
1-\frac{r_{s}}{r}+\frac{f}{2} & 0 & 0 & 0 \\
0 & -1-\frac{r_{s}}{r}+\frac{f}{2} & 0 & 0 \\
0 & 0 & -1-\frac{r_{s}}{r}+\frac{f}{2} & 0 \\
0 & 0 & 0 & -1-\frac{r_{s}}{r}+\frac{f}{2}
\end{array}\right)
$$

\section{SOME OBSERVABLE RESULTS}

At this end let us look for some observable results of the static spherical symmetric solution of the previous section. It is a good idea to see that the extra quantum terms how affect the classical results of the Schwarzschild metric. We present here two effrcts.

1. Light deflection 
As an example consider the light bending effect. Suppose a light ray approaches a star from infinity along the $z$ direction and in the $x-z$ plane with impact parameter $b$. The light ray path would be given by the null geodesic relation, written in the linear field approximation [12]:

$$
d k_{\mu}-\frac{1}{2}\left(\partial_{\mu} h_{\beta}^{\alpha}\right) k_{\alpha} d x^{\beta}=0
$$

where $k_{\mu}$ is the wave number. This equation simplifies to:

$$
d k_{x}=-\frac{\omega}{2} \frac{\partial}{\partial x}\left(-\frac{2 r_{s}}{r}+f\right)_{x=b} d z
$$

where $\omega$ is the frequency of the light beam.

For simplicity, we assume only the term $a_{00}$ contribute in $f$ so we set $^{3}$ :

$$
f=a_{00} j_{0}(\alpha \hbar r)=a_{00} \frac{\sin \alpha \hbar r}{\alpha \hbar r}
$$

Integrating the relation (60), one gets:

$$
\theta \simeq \frac{\int_{-\infty}^{+\infty} d k_{x}}{k_{z}}=-\frac{2 r_{s}}{b}-a_{00}\left(\cos \alpha \hbar b-\frac{\sin \alpha \hbar b}{\alpha \hbar b}\right)
$$

in which $\theta$ is the angle of bending. The first term represents the classical light bending relation, while the second term is the quantum corrections. The dependence of the bending angle on the impact parameter is plotted in the figure (1).

\footnotetext{
${ }^{3}$ If one wants to have a metric not dependent on the spherical angles at infinity, only the coefficients of $Y_{00}$ are nonzero. The behaviour of $j_{0}$ and $n_{0}$ are very similar, so for simplicity we only consider the first one. Considering the second does not change the physics.
} 
As it can be seen, the quantum solution is an oscillating curve around the classical curve. The amplitude of the oscillations is scaled by $a_{00}$, which is an arbitrary constant. So if any oscillatory behaviour be observed in the light bending effect, it can be described by the quantum effects.

\section{Tidal forces}

In order to investigate how the quantum effects change the curvature of the spacetime, it is a good idea to derive the tidal forces. This is a simple task, noting that the tidal forces are in fact the gradient in the gravitational field in locally freely falling frame. In figures (2) and (3) the classical and quantum fieldplots of tidal forces at some point are ploted. Also to see the effect better, in figures (4) and (5) the classical and quantum shape of a sphere falling on a spherically symmetric mass distribution is shown. Note the quantum oscillations in the shape in the last figure.

Acknowledgement The first author wishes to thank Tehran University for supporting this project under the grants provided by the research council.

[1] B.S. De Witt, Phys. Rev., 160, 1113, 1967;

J.A. Wheeler, in Batelle Remcomfres, eds. C. De witt and J.A. Wheeler (Benjamin, New York, 1968).

[2] J.V. Narlikar and T. Padmanabhan, Phys. Rep. 100, 151, 1983;

T. Padmanabhan, Gravitation, Gauge Theories and the Early Universe, eds. B.R. Iyer et.al., 373, (Kluwer, 1989). 
[3] P.R. Holland, The Quantum Theory of Motion, (Cambridge Univ. Press, 1993).

[4] T. Horiguchi, Mod. Phys. Lett. A., 9, 16, 1429, 1994.

[5] F. Shojai and M. Golshani, Int. J. Mod. Phys. A., 13, 4, 677, 1998.

[6] F. Shojai, A. Shojai and M. Golshani, Mod. Phys. Lett. A., 13, 34, 2725, 1998.

[7] F. Shojai, A. Shojai and M. Golshani, Mod. Phys. Lett. A., 13, 36, 2915, 1998.

[8] See e.g. C. Kiefer, Lanl report gr-qc/9906100, in the Proceedings of the Karpacz Winter School on From Cosmology to Quantum Gravity, Springer, 1999;

J.B. Hartle, Quantum Cosmology: Problems for the 21st century, in Physics in the 21st century, edited by K. Kikkawa, H. Kunimoto, H. Ohtsubo, World Scientific, Singapore, 1997.

[9] D. Bohm, Phys. Rev., 85, 166, 1952;

D. Bohm, Phys. Rev., 85, 180, 1952;

D. Bohm and B.J. Hiley, The Undivided Universe, Routledge, 1993.

[10] F. Shojai and M. Golshani, Int. J. Mod. Phys. A., 13, 13, 2135, 1998.

[11] V. Shtanov, Phys. Rev. D54, 4, 1996.

[12] S. Weinberg, Gravitation and Cosmology, (Wiley, New York, 1972);

H.C. Ohanian et.al., Gravitation and Space-time, 2nd edition, WW Norton \& Co., 1994.

[13] A. Shojai, PhD Thesis, Sharif University of Technology, 1997. 


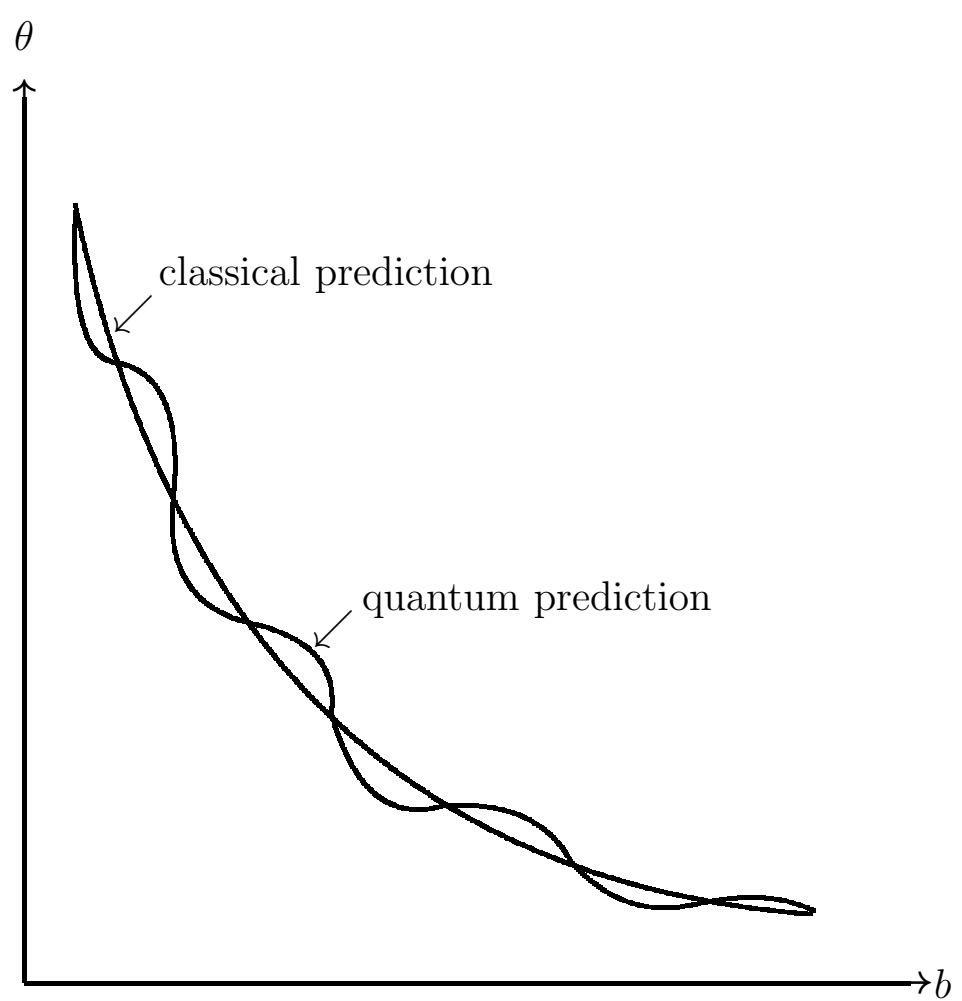

FIG. 1. Classical and quantum light deflection in terms of the impact parameter 


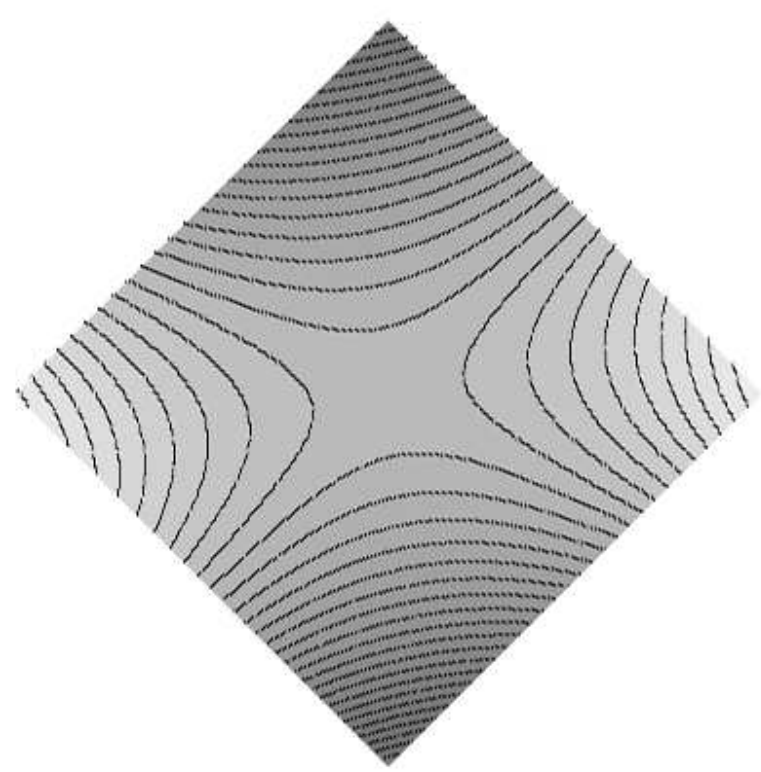

FIG. 2. Classical tidal force contours

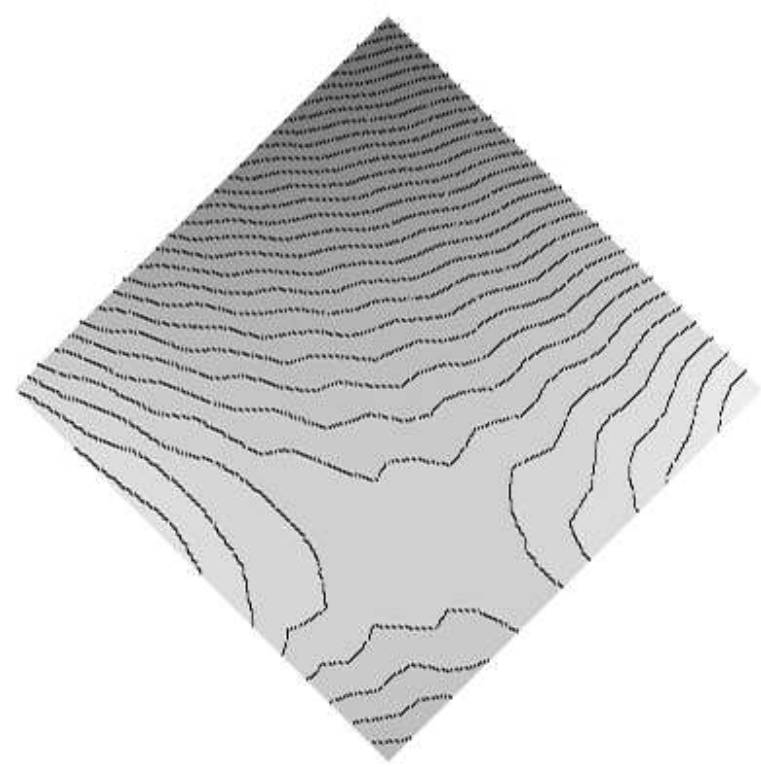

FIG. 3. Quantum tidal force contours 


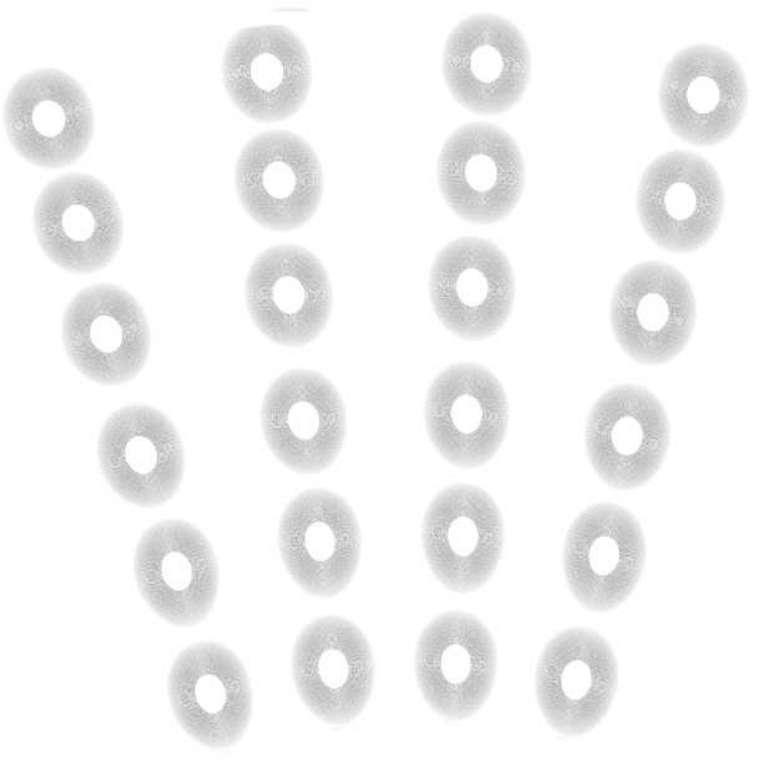

FIG. 4. Classical shape of a freely falling sphere

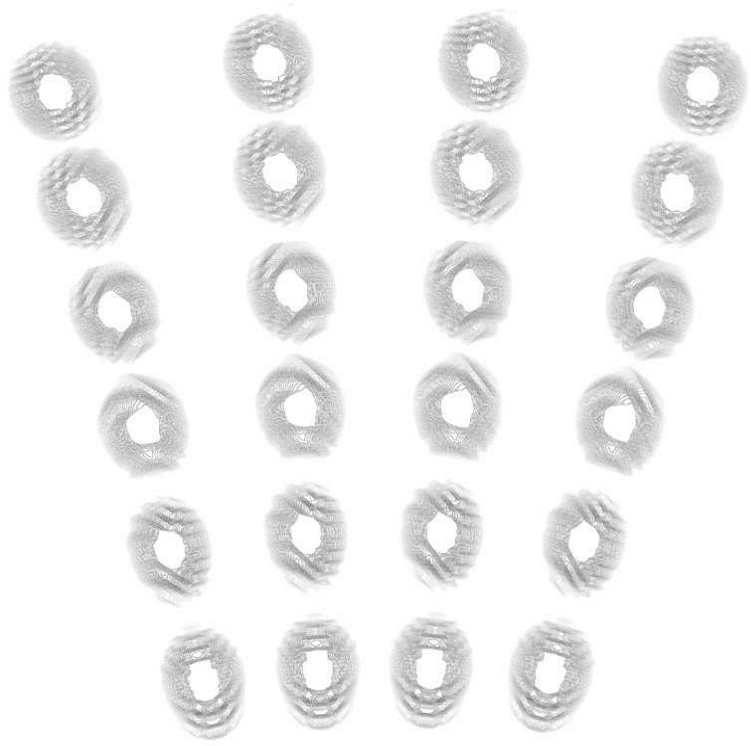

FIG. 5. Quantum shape of a freely falling sphere 